\title{
Do New Guidelines and Technology Make the Routine Pelvic Examination Obsolete?
}

\author{
Carolyn L. Westhoff, M.D., M.Sc., Heidi E. Jones, Ph.D., M.P.H., ${ }^{\star}$ and Maryam Guiahi, M.D.
}

\begin{abstract}
Routine pelvic examinations are the core of the periodic gynecological examination and widely tolerated as a necessary part of health maintenance. Is this examination beneficial for asymptomatic women? Justifications for the pelvic examination include screening for Chlamydia (or gonorrhea) infection, evaluation before initiation of hormonal contraception, screening for cervical cancer, and early detection of ovarian cancer. Current nucleic acid amplification tests for Chlamydia and gonorrhea permit the use of urine and self-administered vaginal swabs, which most women prefer over a pelvic examination. Pelvic examination findings do not affect the decision to prescribe or withhold systemic hormonal contraception; a pelvic examination is not needed to initiate these contraceptives. Recent American College of Obstetricians and Gynecologists (ACOG) guidelines recommend less frequent cervical screening, thus decreasing the frequency of a speculum examination for cervical screening. Bimanual examinations for palpation of the uterus and ovaries are also routinely performed in the United States. Clinical trial data, however, show these examinations do not lead to earlier detection of ovarian cancer. No evidence identifies benefits of a pelvic examination in the early diagnosis of other conditions in the asymptomatic woman. Speculum and bimanual examinations are uncomfortable, disliked by many women, and use scarce time during a well woman visit. Eliminating the speculum examination from most visits and the bimanual examination from all visits of asymptomatic women will free resources to provide services of proven benefit. Overuse of the pelvic examination contributes to high healthcare costs without any compensatory health benefit.
\end{abstract}

\section{Introduction}

I N THE UNITED STATES, the routine pelvic examination is the core of the periodic gynecological examination and widely tolerated by asymptomatic patients who accept it as a necessary part of health maintenance. Elements of the pelvic examination include inspection of the external genitalia and insertion of a vaginal speculum to permit inspection of the upper vagina and cervix and to allow sampling of cells from the cervix. The routine pelvic examination typically includes palpation of the internal pelvic organs with the insertion of two fingers into the vagina accompanied by simultaneous abdominopelvic pressure, known as a bimanual examination. Rectovaginal digital examination may also be performed, particularly for patients over age 40 .

Specific justifications for the pelvic examination include (1) screening for Chlamydia (or gonorrhea) infection, (2) evaluation before the initiation of hormonal contraception, (3) screening for cervical cancer, and (4) early detection of ovarian cancer. We found no detailed information about the number of routine pelvic examinations performed annually in the United States, but according to data from the National Health Interview Survey (NHIS), an estimated 65 million women received a Papanicolaou (Pap) test in 2005. ${ }^{1}$ The performance of a Pap test in the United States may be a reasonable surrogate indicator for the performance of the pelvic examination, often including a bimanual examination and a speculum examination.

Whether or not the routine pelvic examination is beneficial for the asymptomatic woman is unclear. Others have commented on various aspects of the limited value of this examination ${ }^{2-7}$; however, clinical practice in the United States has not abandoned it. This article reviews the value of elements of the pelvic examination as part of the periodic health examination, with particular attention to U.S. evidence-based guidelines and the role of newer technologies. We also consider the potential harms of pelvic examinations in asymptomatic women. The role of the pelvic examination in evaluation of women with symptoms or for follow-up of women with known abnormalities is a separate topic and is not considered here. 


\section{Role of the Pelvic Examination for Sexually Transmitted Infection Screening}

The U.S. Preventive Task Services Force (USPSTF), the Centers for Disease Control and Prevention (CDC), and other professional organizations recommend routine periodic screening for Chlamydia for all sexually active women $<26$ years and for older women with higher than average risk factors. ${ }^{8,9}$ Routine screening for Chlamydia in young women is based on 1996 data showing that screening decreases the incidence of pelvic inflammatory disease (PID). ${ }^{10}$ Although similar data for gonorrhea are lacking, combined screening is simple, widely used, and may be beneficial in communities with a high incidence of gonorrhea. Annual Chlamydia screening is now covered by health plans and is often used as a benchmark to assess quality of care. ${ }^{11}$

Until the 1980s, cell culture techniques were required to identify Chlamydia; thus, screening was expensive and not readily available. Because Chlamydia is an obligate intracellular organism, testing with cell cultures depended on obtaining endocervical cell specimens directly from the cervix after first wiping away excess mucus. Today's new technology, highly sensitive and specific nucleic acid amplification tests (NAATs), permits the use of urine and self-collected vaginal swabs for testing for Chlamydia in women. Selfadministered vaginal swabs are at least equal to clinicianobtained swabs in sensitivity, with no difference in specificity for Chlamydia. ${ }^{12}$ Most patients prefer self-administered vaginal swabs over a pelvic examination. ${ }^{12-14}$ The same specimens (urine or vaginal self-swab) are also adequate to test for gonorrhea. ${ }^{15}$ Both urine and vaginal swabs for Chlamydia and gonorrhea screening are more cost-effective and cost-saving than clinician-collected specimens during a pelvic examination. $^{16,17}$ Thus, new highly sensitive tests with urine or selfobtained vaginal swabs obviate the need for vaginal speculum examination to screen for infection of the cervix in asymptomatic women. In 2009, the CDC recommended the use of NAATs with vaginal self-swabs as the preferred Chlamydia screening test for women; female urine tests are acceptable but are not quite as good as genital swabs. ${ }^{18}$ Home tests for Chlamydia screening kits are currently available in the United Kingdom via the Internet ${ }^{19}$ and at retail pharmacies. At this time, such home test kits are being evaluated in the United States but are not yet commercially available. ${ }^{20}$

\section{Role of the Pelvic Examination for Provision of Contraception}

Among young women in the United States, a pelvic examination frequently may take place before initiation of systemic hormonal contraception. A pelvic examination is certainly needed to fit a contraceptive diaphragm or to insert an intrauterine device (IUD); however, no findings from a pelvic examination would change the decision to prescribe systemic hormonal contraception. Therefore, guidelines from the U.S. Food and Drug Administration (FDA), World Health Organization (WHO), American College of Obstetricians and Gynecologist (ACOG), and Planned Parenthood Federation of America (PPFA) explicitly specify that a pelvic examination is not required in an asymptomatic woman for the initiation of systemic hormonal contraceptives. ${ }^{21-24}$ Women in the age group most commonly starting hormonal contraceptives do need screening for Chlamydia, which can be accomplished with either collection of urine or a self-administered vaginal swab. In contrast, many of these young women will not yet need cervical cancer screening; the most recent ACOG guidelines do not recommend cervical cancer screening before the age of $21 .^{25}$ Therefore, systemic hormonal contraceptives can be initiated more quickly and easily by eliminating the need for a pelvic examination in women under $21 .{ }^{5}$ Of course, cervical screening and other preventive services in women using hormonal contraception should continue to follow national guidelines, but timing of these services does not need to be linked to the initiation or the refill of contraceptives. Unintended pregnancy remains an epidemic problem, ${ }^{26}$ and the need for cervical screening should never delay contraceptive protection.

\section{Role of the Pelvic Examination for Cervical Cancer Screening}

Perhaps the most common indication for a vaginal speculum examination is to obtain a specimen for cervical cancer screening using cytology, which is carried out at least 60 million times per year in the United States. ${ }^{1}$ Annual cervical screening for all adult women has been routine care in the United States for several decades. The 2009 ACOG Practice Bulletin, however, recommends a decrease in the frequency of cytological screening: women should begin to undergo screening for the precursors of cervical cancer starting at age 21 regardless of the age of sexual debut. ${ }^{25}$ Screening should then occur approximately every 2 years until age 29. For women aged 30 to about 65 , screening can take place every 3 years. If a woman aged $\geq 30$ has simultaneous adjunctive screening for human papillomavirus (HPV), United Kingdom and European data show that the risk of developing a highgrade cervical lesion is markedly reduced for at least 4-6 years as long as both tests are negative. ${ }^{27,28}$ For any women with abnormal screening results, of course, follow-up testing will be more frequent and will need to include visualization of the cervix. $^{29}$ The ACOG recommendations for less frequent cervical cancer screening markedly decrease the number of times a clinician needs to carry out a speculum examination in order to sample the cervix, and they clarify that during cervical screening, a bimanual examination is not needed.

Future changes in testing will have an even greater impact on the need for direct clinician-obtained samples from the cervix. Primary screening with a DNA amplification test for oncogenic strains of HPV is more sensitive (and only slightly less specific) for high-grade dysplasia than primary screening using cytology. ${ }^{28,30-32}$ Because a self-administered vaginal swab produces a specimen that is adequate for HPV testing, 33,34 the speculum examination for obtaining a cervical specimen may become unnecessary for asymptomatic women $>$ age 30 . Among women aged 21-29, however, transient HPV infection is prevalent ${ }^{35}$; therefore, primary HPV screening is less useful, and cervical cytology (needing a speculum examination every 2 years) may remain the preferred screening test in that age group.

Until primary HPV testing becomes routine ${ }^{36}$ or until we have a satisfactory approach to self-sampling for cytology, women will still need occasional speculum examinations for cervical screening. Nevertheless, the frequency of these examinations can be less than many patients currently expect. Following current guidelines, women need 4-5 speculum 
examinations for cervical screening when in their 20s and perhaps an additional 10 examinations in their 30s-60s, in contrast to the annual test that many patients currently receive. Of course, women who have an abnormal test result need more frequent follow-up. Women may prefer less frequent testing for its own sake and will also benefit from fewer false positive results ${ }^{37}$; thus, they will also experience fewer consequent diagnostic and treatment procedures.

\section{Role of the Pelvic Examination for Detection of Ovarian Cancer}

In the United States, the speculum examination is routinely accompanied by a bimanual pelvic examination for palpation of the uterus and ovaries in hopes that this will lead to earlier detection of ovarian cancer. Unfortunately, bimanual examinations do not lead to earlier detection of ovarian cancer. ${ }^{38-41}$ A comparison of the United Kingdom, where bimanual examination is not routinely performed, and the United States, where bimanual examination is routine and frequent, showed no difference in the proportion of women diagnosed with ovarian cancer at stage I. ${ }^{42}$ Bimanual examination has poor sensitivity and specificity for the detection of ovarian cancer. In an Australian case series, none of the women with an adnexal mass detected by bimanual examination had ovarian cancer. ${ }^{43}$ The use of the bimanual examination for identifying the postmenopausal palpable ovary for early detection of ovarian cancer has been abandoned, and the use of transvaginal sonography is preferred in the symptomatic patient. ${ }^{44-51}$ Most recently, the Prostate, Lung, Colorectal, and Ovarian Cancer Screening Trial of the National Cancer Institute (NCI) eliminated the bimanual examination portion of screening after review of the data determined that no ovarian cancers had been detected with this modality alone. ${ }^{52}$ Guidelines from the USPSTF, the American Cancer Society (ACS), and ACOG do not recommend the bimanual examination for early detection of ovarian cancer. ${ }^{53}$

\section{Are There Benefits from Pelvic Examinations in Asymptomatic Women?}

Even in asymptomatic women, the bimanual pelvic examination is an opportunity for diagnosing uterine fibroids and ovarian cysts or other lesions, although few studies are available to assess the value of diagnosis in asymptomatic women. In an audit of 420 asymptomatic women attending an Australian sexual health clinic, 93\% had no abnormality found by pelvic examination. The other $7 \%$ had abnormalities, such as bacterial vaginosis and yeast; these conditions may resolve spontaneously, and a delay in diagnosis among asymptomatic women does not lead to harm. ${ }^{2}$ Uterine fibroids and ovarian cysts may be contributory in a woman who has pelvic pain, distention, menstrual difficulties, or infertility, but we found no evidence that diagnosis of fibroids before development of symptoms will lead to a clinical benefit. In general, the clinical approach to asymptomatic palpable fibroids includes the use of imaging studies and more frequent follow-up examinations until such time that development of symptoms (if ever) actually prompts an intervention. ${ }^{54}$ For asymptomatic ovarian cysts, a possible benefit of early excision would be to preclude an emergency operation caused by torsion or rupture of the cyst; however, these complications are rare, and operative outcomes are good. ${ }^{55}$
Thus, prophylactic surgery to excise an asymptomatic ovarian cyst would benefit a few women by avoiding an ill-timed emergency procedure. For each such woman, however, many additional women will undergo surgical excision of a benign cyst that would never have caused symptoms.

What about other cancers? The rectovaginal examination was once recommended for rectal cancer screening. Current recommendations are to use sigmoidoscopy, colonoscopy, or fecal sample testing for colorectal cancer screening. ${ }^{56}$ Thus, the rectovaginal examination should be limited to use in the evaluation of symptoms. There is currently no practice of screening for vaginal or vulvar neoplasms in the general population, and we found no published assessments of whether early detection of these rare lesions would be feasible or would change clinical outcomes. Postmenopausal or other abnormal bleeding is the harbinger of uterine cancer; for women without symptoms, there are no screening tests for uterine cancer.

\section{Are There Adverse Consequences of Routine Pelvic Examinations?}

In contrast to the women who avoid pelvic examinations, other women are firmly persuaded of the necessity of regular, often annual, examinations. A clinical challenge is to adopt new cervical screening guidelines that call for less frequent testing. Overscreening leads to false positive tests, which in turn lead to additional diagnostic evaluation with attendant discomfort, costs, and risks. These risks are particularly salient among young women, who have a high prevalence of nonprogressive dysplasia. Such young women have the most to lose from aggressive evaluation and treatment, particularly conization of the cervix. The risk of preterm birth consequent to treatment for high-grade dysplasia ${ }^{57}$ is likely to affect their obstetrical future, even while the risk of progression to cervical cancer is exceedingly small. A key impetus for the ACOG recommendations for less screening among young women is that surgical treatments for dysplasia are associated with a 2-fold increased risk of subsequent premature birth and with additional adverse obstetrical outcomes. ${ }^{57-59}$

Many speculum examinations for cervical screening are accompanied by bimanual examinations, which we have shown are unnecessary in asymptomatic women. Findings on a bimanual examination, even in a completely asymptomatic woman, will often lead to additional diagnostic evaluation, such as sonography, and often to surgery for excision of asymptomatic cysts and fibroids that may have never become clinically apparent. Frequent, routine bimanual examinations may partly explain why U.S. rates of ovarian cystectomy and hysterectomy are more than twice as high as rates in European countries, where the use of the pelvic examination is limited to symptomatic women. ${ }^{42,60}$

\section{Do Women Avoid the Pelvic Examination?}

The ritual of a routine pelvic examination can act as a barrier to needed care as a result of fear, embarrassment, discomfort, and inconvenience. ${ }^{60-62}$ Numerous studies show that adolescents delay initiation of needed contraception because of fear of the pelvic examination. ${ }^{5}$ Similarly, avoidance of this examination may be a reason that only about $50 \%$ of insured women aged $<26$ undergo recommended annual screening for Chlamydia. ${ }^{63}$ The expectation of the pelvic 
examination can thus obstruct public health efforts to reduce unwanted pregnancy in the United States as well as efforts to prevent infertility through early treatment of Chlamydia infection. ${ }^{64}$ Relevant guidelines from ACOG recommend that adolescents do not need routine pelvic examinations. ${ }^{65}$ Providers need to ensure their patients know they can initiate contraceptives and receive screening and treatment for STIs without a pelvic examination.

Over $50 \%$ of cervical cancer cases among privately insured women occur among those who have not been screened in the past 3 years despite having access to screening. ${ }^{66}$ Even insured women experience barriers to care; concerns about the pelvic examination may partially explain the lack of screening in these women. A recent study in the Netherlands evaluated over 28,000 women who had missed multiple invitations for cervical screening; $27 \%$ of these women accepted screening using a self-lavaging device that eliminated the need for a speculum examination. ${ }^{67}$ This finding suggests that cervical screening coverage may improve when we adopt primary HPV testing with self-sampling as an alternative to the current speculum examination.

\section{How Do Other Countries Use the Pelvic Examination?}

In Sweden, the Netherlands, and the United Kingdom, the bimanual examination is performed only in the evaluation of women with symptoms. It is not combined with routine cervical screening and is not a part of the periodic examination of asymptomatic women. ${ }^{68}$ This practice difference may explain, in part, why women in the United Kingdom undergo so much less ovarian cystectomy and hysterectomy than do women in the United States. ${ }^{42,69}$

In Australia, use of the pelvic examination is more similar to that in the United States. Two Australian general practitioners reviewed the literature about routine pelvic examinations, and found no evidence to support this practice. ${ }^{7}$ The authors then carried out interviews with 27 Australian general practitioners to explore why they perform these examinations. The physicians generally agreed that the value of a screening pelvic examination was questionable and reported performing such examinations for reasons including patient reassurance, documenting the norm, "because I was taught to," for legal reasons, and for completeness. ${ }^{6}$ To our knowledge, no U.S. data examine physician attitudes to performing a routine pelvic examination.

\section{Conclusions}

We have shown that the pelvic examination in asymptomatic women is not needed to screen for STIs, is not needed to initiate hormonal contraception, and is not beneficial for early detection of ovarian cancer. The speculum examination is now needed every 2-3 years for cervical screening and, with the advent of primary HPV testing with self-collected specimens, may be needed even less often for screening purposes in the future. Women's avoidance of the healthcare system because of dislike of the pelvic examination directly interferes with public health goals of screening for Chlamydia to reduce infertility, reducing unintended pregnancy, and increasing appropriate cervical cancer screening coverage.

We do not know what fraction of excessive cervical excisions, conizations, ovarian cystectomies, and hysterectomies is consequent to unnecessary examinations. Nevertheless, we do know that substantial resources are consumed every year in the simple performance of millions of pelvic examinations in asymptomatic women, even while clinicians lack time to provide other proven preventive services. In presenting their new cervical screening guidelines, ACOG states that "annual gynecologic examinations may still be appropriate even if cervical cytology is not performed at each visit." ${ }^{25}$ This statement implies its converse: that an annual gynecological examination may not be appropriate. Eliminating unneeded annual pelvic examinations will please many women and simultaneously free their physicians to provide other more needed care.

Overutilization of services is the most important contributor to the high cost of U.S. healthcare. ${ }^{70}$ Increasingly, we recognize that more services do not always lead to improved health outcomes and that often the opposite is true: that more services are associated with worse heath outcomes. ${ }^{71}$ The routine pelvic examination may be an example of more service leading to worse outcomes. It is time to get asymptomatic women off the table; we need to perform speculum examinations less frequently and completely do away with the bimanual examination in asymptomatic women, as is already routine practice in many European settings.

\section{Disclosure Statement}

C.L.W. and H.E.J. received research support from Delphi Biosciences BV (Scherpenzeel, Netherlands). The authors have no other conflicts of interest to declare.

\section{References}

1. Eltoum IA, Roberson J. Impact of HPV testing, HPV vaccine development, and changing screening frequency on national Pap test volume: Projections from the National Health Interview Survey (NHIS). Cancer 2007;111:34-40.

2. Lee DM, Chen MY, Bradshaw CS, Fairley CK. Is routine vaginal examination necessary for asymptomatic women attending sexual health services? Int J STD AIDS 2006;17:631-632.

3. Schachter J, Shafer MA, Young M, Ott M. Routine pelvic examinations in asymptomatic young women. N Engl J Med 1996;335:1847-1848.

4. Shulman LP. New recommendations for the periodic wellwoman visit: Impact on counseling. Contraception 2006;73:319-324.

5. Stewart FH, Harper CC, Ellertson CE, Grimes DA, Sawaya GF, Trussell J. Clinical breast and pelvic examination requirements for hormonal contraception: Current practice vs. evidence. JAMA 2001;285:2232-2239.

6. Stewart RA, Thistlethwaite J, Evans R. Pelvic examination of asymptomatic women-Attitudes and clinical practice. Aust Fam Physician 2008;37:493-496.

7. Stewart RA, Thistlethwaite J. Routine pelvic examination for asymptomatic women-Exploring the evidence. Aust Fam Physician 2006;35:873-877.

8. Screening for chlamydial infection: U.S. Preventive Services Task Force recommendation statement. Ann Intern Med 2007;147:128-134.

9. Workowski KA, Berman SM. Sexually transmitted diseases treatment guidelines, 2006. MMWR Recomm Rep 2006;55: $1-94$.

10. Scholes D, Stergachis A, Heidrich FE, Andrilla H, Holmes KK, Stamm WE. Prevention of pelvic inflammatory disease 
by screening for cervical chlamydial infection. $N$ Engl J Med 1996;334:1362-1366.

11. Chlamydia screening among sexually active young female enrollees of health plans-United States, 2000-2007. MMWR 2009;58:362-365.

12. Schachter J, Chernesky MA, Willis DE, et al. Vaginal swabs are the specimens of choice when screening for Chlamydia trachomatis and Neisseria gonorrhoeae: Results from a multicenter evaluation of the APTIMA assays for both infections. Sex Transm Dis 2005;32:725-728.

13. Chernesky MA, Hook EW 3rd, Martin DH, et al. Women find it easy and prefer to collect their own vaginal swabs to diagnose Chlamydia trachomatis or Neisseria gonorrhoeae infections. Sex Transm Dis 2005;32:729-733.

14. Hobbs MM, van der Pol B, Totten $\mathrm{P}$, et al. From the NIH: Proceedings of a Workshop on the Importance of SelfObtained Vaginal Specimens for Detection of Sexually Transmitted Infections. Sex Transm Dis 2008;35:8-13.

15. Hook EW 3rd, Ching SF, Stephens J, Hardy KF, Smith $\mathrm{KR}$, Lee HH. Diagnosis of Neisseria gonorrhoeae infections in women by using the ligase chain reaction on patient-obtained vaginal swabs. J Clin Microbiol 1997;35: 2129-2132.

16. Shafer MA, Pantell RH, Schachter J. Is the routine pelvic examination needed with the advent of urine-based screening for sexually transmitted diseases? Arch Pediatr Adolesc Med 1999;153:119-125.

17. Blake DR, Maldeis N, Barnes MR, Hardick A, Quinn TC, Gaydos CA. Cost-effectiveness of screening strategies for Chlamydia trachomatis using cervical swabs, urine, and selfobtained vaginal swabs in a sexually transmitted disease clinic setting. Sex Transm Dis 2008;35:649-655.

18. Association of Public Health Laboratories \& Centers for Disease Control and Prevention. Laboratory diagnostic testing for Chlamydia trachomatis and Neisseria gonorrhoeae. Expert Consultation Meeting Summary Report, 2009.

19. Preventx. Could you have Chlamydia? Available at www.preventx.com/ Accessed June 14, 2010.

20. Gaydos CA, Dwyer K, Barnes M, et al. Internet-based screening for Chlamydia trachomatis to reach nonclinic populations with mailed self-administered vaginal swabs. Sex Transm Dis 2006;33:451-457.

21. World Health Organization. Department of Reproductive Health and Research. Improving access to quality care in family planning: Medical eligibility criteria for contraceptive use, 2nd ed. Geneva: WHO, 2000.

22. U.S. Food and Drug Administration. Labeling guidance text for combination oral contraceptives, prescribing information, physician labeling, 1994 (Docket number: 2000D-1350). Available at www.fda.gov/ohrms/dockets/dailys/04/may04/ 050604/050604.htm Accessed January 4, 2010.

23. Planned Parenthood Federation of America. Manual of medical standards and guidelines: Hormonal contraceptive use. New York: PPFA National Medical Commitee; 1999.

24. American College of Obstetricians and Gynecologists. Educational bulletin: Oral contraceptives for adolescents: Benefits and safety in health care for adolescents. Washington, DC: ACOG, 2003.

25. American College of Obstetricians and Gynecologists. ACOG practice bulletin No. 109: Cervical cytology screening. Obstet Gynecol 2009;114:1409-1420.

26. An overview of abortion in the United States, 2005. Available at www.guttmacher.org/media/presskits/2005/06/28/ abortionoverview.html Accessed January 5, 2010.
27. Cuzick J, Szarewski A, Mesher D, et al. Long-term follow-up of cervical abnormalities among women screened by HPV testing and cytology-Results from the Hammersmith Study. Int J Cancer 2008;122:2294-2300.

28. Bulkmans NW, Berkhof J, Rozendaal L, et al. Human papillomavirus DNA testing for the detection of cervical intraepithelial neoplasia grade 3 and cancer: 5-year follow-up of a randomised controlled implementation trial. Lancet 2007;370:1764-1772.

29. American Society for Colposcopy and Cervical Pathology (ASCCP). 2006 consensus guidelines for the management of women with abnormal cervical cancer screening tests. J Lower Gen Tract Dis 2007;11:201-222.

30. Mayrand MH, Duarte-Franco E, Rodrigues I, et al. Human papillomavirus DNA versus Papanicolaou screening tests for cervical cancer. N Engl J Med 2007;357:1579-1588.

31. Ronco G, Segnan N. HPV testing for primary cervical cancer screening. Lancet 2007;370:1740-1742.

32. Ronco G, Giorgi-Rossi P, Carozzi F, et al. Efficacy of human papillomavirus testing for the detection of invasive cervical cancers and cervical intraepithelial neoplasia: A randomised controlled trial. Lancet Oncol 2010;11:249-257.

33. Ogilvie GS, Patrick DM, Schulzer M, et al. Diagnostic accuracy of self-collected vaginal specimens for human papillomavirus compared to clinician-collected human papillomavirus specimens: A meta-analysis. Sex Transm Infect 2005;81:207-212.

34. Petignat P, Faltin DL, Bruchim I, Tramer MR, Franco EL, Coutlee F. Are self-collected samples comparable to physician-collected cervical specimens for human papillomavirus DNA testing? A systematic review and meta-analysis. Gynecol Oncol 2007;105:530-535.

35. Ho GY, Bierman R, Beardsley L, Chang CJ, Burk RD. Natural history of cervicovaginal papillomavirus infection in young women. N Engl J Med 1998;338:423-428.

36. Wright TC Jr. Cervical cancer screening in the 21st century: Is it time to retire the Pap smear? Clin Obstet Gynecol 2007;50:313-323.

37. Insinga RP, Glass AG, Myers ER, Rush BB. Abnormal outcomes following cervical cancer screening: Event duration and health utility loss. Med Decis Making 2007;27:414422.

38. Padilla LA, Radosevich DM, Milad MP. Limitations of the pelvic examination for evaluation of the female pelvic organs. Int J Gynaecol Obstet 2005;88:84-88.

39. Padilla LA, Radosevich DM, Milad MP. Accuracy of the pelvic examination in detecting adnexal masses. Obstet Gynecol 2000;96:593-598.

40. Bass KM, Westhoff C, Bush TL. Ovarian cancer: Epidemiologic and clinical perspectives and the feasibility of screening. Menopause 1995;2:145-158.

41. Smith LH, Oi RH. Detection of malignant ovarian neoplasms: A review of the literature. I. Detection of the patient at risk; clinical, radiological and cytological detection. Obstet Gynecol Surv 1984;39:313-328.

42. Westhoff C, Clark CJ. Benign ovarian cysts in England and Wales and in the United States. Br J Obstet Gynaecol 1992;99:329-332.

43. Grover SR, Quinn MA. Is there any value in bimanual pelvic examination as a screening test. Med J Aust 1995;162:408410.

44. U.S. Preventive Services Task Force. Screening for ovarian cancer: Recommendation statement. Am Fam Physician 2005;71:759-762. 
45. Ueland FR, Depriest PD, Desimone CP, et al. The accuracy of examination under anesthesia and transvaginal sonography in evaluating ovarian size. Gynecol Oncol 2005;99:400-403.

46. Barber HR, Graber EA. The PMPO syndrome (postmenopausal palpable ovary syndrome). CA Cancer J Clin 1972; 22:357-359.

47. American College of Obstetricians and Gynecologists. ACOG committee opinion No. 280, December 2002. The role of the generalist obstetrician-gynecologist in the early detection of ovarian cancer. Obstet Gynecol 2002;100:14131416.

48. Reuss ML, Kolton S, Tharakan T. Transvaginal ultrasonography in gynecologic office practice: Assessment in 663 premenopausal women. Am J Obstet Gynecol 1996;175: 1189-1194.

49. Ferrini R. Screening asymptomatic women for ovarian cancer: American College of Preventive Medicine practice policy. Am J Prev Med 1997;13:444-446.

50. American College of Physicians. Screening for ovarian cancer: Recommendations and rationale. Ann Intern Med 1994;121:141-142.

51. National Institute of Health (1994). Ovarian cancer: Screening, treatment and follow-up. NIH Consensus Statement 12: 16-17.

52. Buys SS, Partridge E, Greene $\mathrm{MH}$, et al. Ovarian cancer screening in the Prostate, Lung, Colorectal and Ovarian (PLCO) Cancer Screening Trial: Findings from the initial screen of a randomized trial. Am J Obstet Gynecol 2005; 193:1630-1639.

53. Clarke-Pearson DL. Clinical practice. Screening for ovarian cancer. N Engl J Med 2009;361:170-177.

54. American College of Obstetricians and Gynecologists. ACOG practice bulletin. Alternatives to hysterectomy in the management of leiomyomas. Obstet Gynecol 2008;112:387-400.

55. White M, Stella J. Ovarian torsion: 10-year perspective. Emerg Med Australas 2005;17:231-237.

56. Smith RA, Cokkinides V, Brooks D, Saslow D, Brawley OW. Cancer screening in the United States, 2010: A review of current American Cancer Society guidelines and issues in cancer screening. CA Cancer J Clin 2010;60:99-119.

57. Albrechtsen S, Rasmussen S, Thoresen S, Irgens LM, Iversen OE. Pregnancy outcome in women before and after cervical conisation: Population-based cohort study. BMJ 2008;337: a1343.

58. Samson SL, Bentley JR, Fahey TJ, McKay DJ, Gill GH. The effect of loop electrosurgical excision procedure on future pregnancy outcome. Obstet Gynecol 2005;105:325-332.

59. Noehr B, Jensen A, Frederiksen K, Tabor A, Kjaer SK. Loop electrosurgical excision of the cervix and subsequent risk for spontaneous preterm delivery: A population-based study of singleton deliveries during a 9-year period. Am J Obstet Gynecol 2009;201:e31-36.
60. Knops-Dullens T, de Vries N, de Vries H. Reasons for nonattendance in cervical cancer screening programmes: An application of the Integrated Model for Behavioural Change. Eur J Cancer Prev 2007;16:436-445.

61. Millstein SG, Adler NE, Irwin CE Jr. Sources of anxiety about pelvic examinations among adolescent females. J Adolesc Health Care 1984;5:105-111.

62. Armstrong KA, Stover MA. SMART START: An option for adolescents to delay the pelvic examination and blood work in family planning clinics. J Adolesc Health 1994;15:389-395.

63. United States Centers for Disease Control and Prevention. Chlamydia screening among sexually active young female enrollees of health plans-United States, 2000-2007. MMWR 2009;58:362-365.

64. United States Centers for Disease Control and Prevention. Sexually transmitted disease surveillance 2007 supplement: Chlamydia prevalence monitoring project annual report 2007. Atlanta, GA: U.S. Department of Health and Human Services, Centers for Disease Control and Prevntion, 2009.

65. American College of Obstetricians and Gynecologists. Guidelines for women's health care: A resource manual, 3rd ed. Washington, DC: ACOG, 2007.

66. Leyden WA, Manos MM, Geiger AM, et al. Cervical cancer in women with comprehensive health care access: Attributable factors in the screening process. J Natl Cancer Inst 2005;97:675-683.

67. Gok M, Heideman DA, van Kemenade FJ, et al. HPV testing on self-collected cervicovaginal lavage specimens as screening method for women who do not attend cervical screening: Cohort study. BMJ 2010;340:c1040.

68. Cromer BA, McCarthy M. Family planning services in adolescent pregnancy prevention: The views of key informants in four countries. Fam Plann Perspect 1999;31:287-293.

69. McPherson K, Wennberg JE, Hovind OB, Clifford P. Smallarea variations in the use of common surgical procedures: An international comparison of New England, England, and Norway. N Engl J Med 1982;307:1310-1314.

70. Emanuel EJ, Fuchs VR. The perfect storm of overutilization. JAMA 2008;299:2789-2791.

71. Grady D, Redberg RF. Less is more: How less health care can result in better health. Arch Intern Med 2010;170:749750 .

Address correspondence to:

Carolyn L. Westhoff, M.D.

Columbia University Medical Center 622 W. 168th Street New York, NY 10032

E-mail: clw3@columbia.edu 